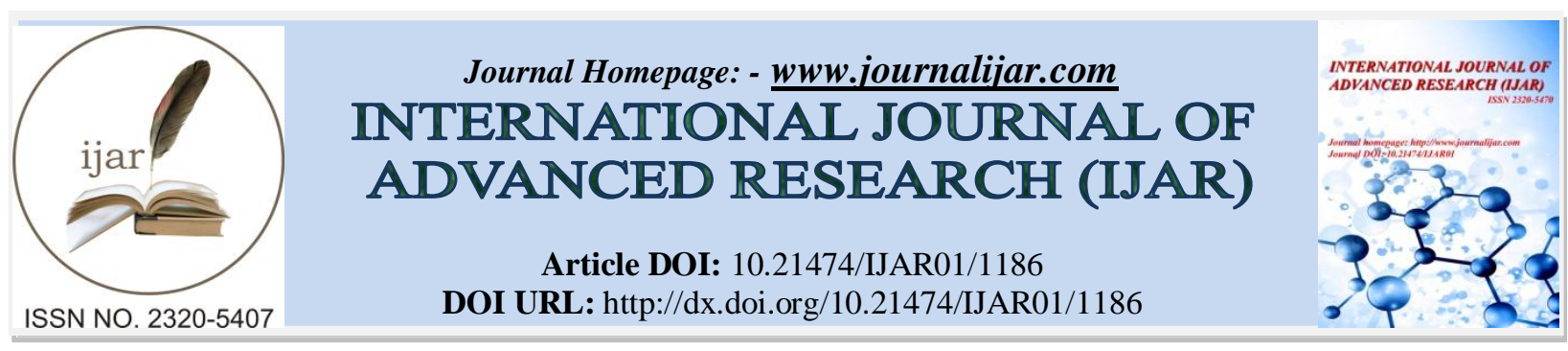

RESEARCH ARTICLE

\title{
EMPIRICAL MUTUAL CORRELATION FORMULA FOR SEASONAL IONOSPHERIC PARAMETERS VARIATION OVER MIDDLE EAST REGION.
}

Marwa D. Abdulkareem* and Khalid A. Hadi.

Department of astronomy and space, college of science, university of baghdad, baghdad, iraq.

\section{Manuscript Info}

Manuscript History

Received: 12 June 2016

Final Accepted: 13 July 2016

Published: August 2016

Key words:-

Ionospheric Parameters, HF Radio Wave

Propagation, Sky Wave Propagation

\section{Abstract}

In this project, the behavior of ionospheric parameters has been studied for the seasonal times of the years (2009 \& 2014) of solar cycle 24.A mutual correlation formula between the ionospheric parameters, maximum usable frequency (MUF), optimum workable frequency (OWF) \& lowest usable frequency (LUF) have been suggested for the communication links distributed over Middle East Region. The values of the MUF and OWF parameters were generated from the international communication $\mathrm{HF}$ model (ASAPS) for the Minimum and Maximum of solar cycle 24, While the LUF parameter was generated using the (REC533) international communication model. The tested correlation coefficients between these parameters showed a good correlation with each other. The suggested formula gave a good fitting between the present values and the generated data of the international model and the international recommended criterion (theoretical).

Copy Right, IJAR, 2016,. All rights reserved.

\section{Introduction:-}

In the Earth's atmosphere, the ionosphere is the part of the Earth's upper atmosphere that makes up less than one percent of the mass of the atmosphere above $50 \mathrm{~km}$. It extends from about $50 \mathrm{~km}$ altitude to about $1000 \mathrm{~km}$ and more where it merges with near Earth space environment. The ionosphere contains charged particles due to intense UV radiations from the sun.In the day there may be four regions present called the D, E, F1 (lower) and F2 (upper) regions. At night the D, E and F1 regions become very much depleted of free electrons, leaving only the F2 region available for communications [1]. The Radio signals affects by the ionosphere in different ways depending on their frequencies. On frequencies below about $30 \mathrm{MHz}$ the ionosphere may act as an efficient reflector, allowing radio communication to distances of many thousands of kilometers [2]. Radio signals on frequencies above $30 \mathrm{MHz}$ usually penetrate the ionosphere and, therefore, are useful for ground-to-space communications. High frequency ( 3 to $30 \mathrm{MHz}$ ) radio signals can propagate to a distant receiver. Figure (1), via the following methods [3]: Propagation is by ground, direct and sky wave.

Corresponding Author:- Marwa D. Abdulkareem.

Address:- Department of Astronomy and Space, College of Science, University of Baghdad, Baghdad, Iraq. 


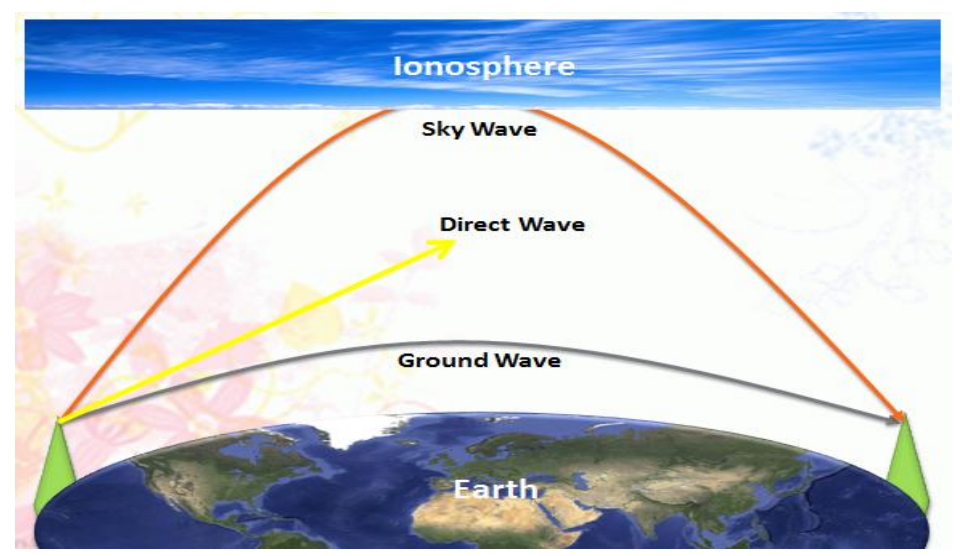

Figure 1:- Types of HF propagation.

The Ionospheric Communication Parameters:-

The ionospheric parameters are defined as the best operation frequencies that are propagated through ionosphere, so these parameters can be defined as:-

Maximum Usable Frequency (MUF) is the highest ionospheric frequency which can be used to maintain successful communication link only $50 \%$ of the time over a particular path under given ionospheric conditions between two sits.

Optimum Working Frequency (OWF) is the optimum reliable frequency can be reflected from ionosphere layer that is worked only $90 \%$ for specific time [4].

Lowest Usable Frequency (LUF) is the lower frequency that allows reliable long-range HF radio communication between two points by ionospheric refraction that is worked only $10 \%$ of the days of the month at a given time of day on a specified path [5].

The above parameters are greatly changed with several factors like solar cycle, spatial and temporal scales that have strong influence on the state of the ionosphere layer. [6].

\section{The International Communication Model:-}

The dataset of the required ionospheric parameters over the Middle East region have been calculated using the REC533 and ASAPS international models. The REC533 propagation prediction model which used for estimating the reliability and compatibility between frequencies of about $3-30 \mathrm{MHz}$. This implementation represents one of the modern radio broadcasting versions of ITU. ASAPS model which considered as one of the most accurate and advanced HF sky wave propagation models allow the prediction of Sky Wave communication conditions in the HF radio spectrum or Short Wave Band (1 to 45 $\mathrm{MHz}$ ) that based on an Ionospheric model, developed by IPS Radio and Space Services of the Australian Bureau of Meteorology and ITU-R / CCIR models.

\section{Datasets Analysis:-}

The variation and behavior of the ionospheric parameters have been studied in order to get the mutual correlation between these parameters for the seasonal time of the years 2009 \& 2014 of solar cycle 24 between HF communication terminals that distributed over Middle East Zone. Baghdad city $\left(44.42^{\circ} \mathrm{E}\right.$, $33.32^{\circ} \mathrm{N}$ ) has been select to represent a transmitter station while sixty-five locations that spread over Middle East Zone have been considered as a receiver station, as shown in figure (2). 


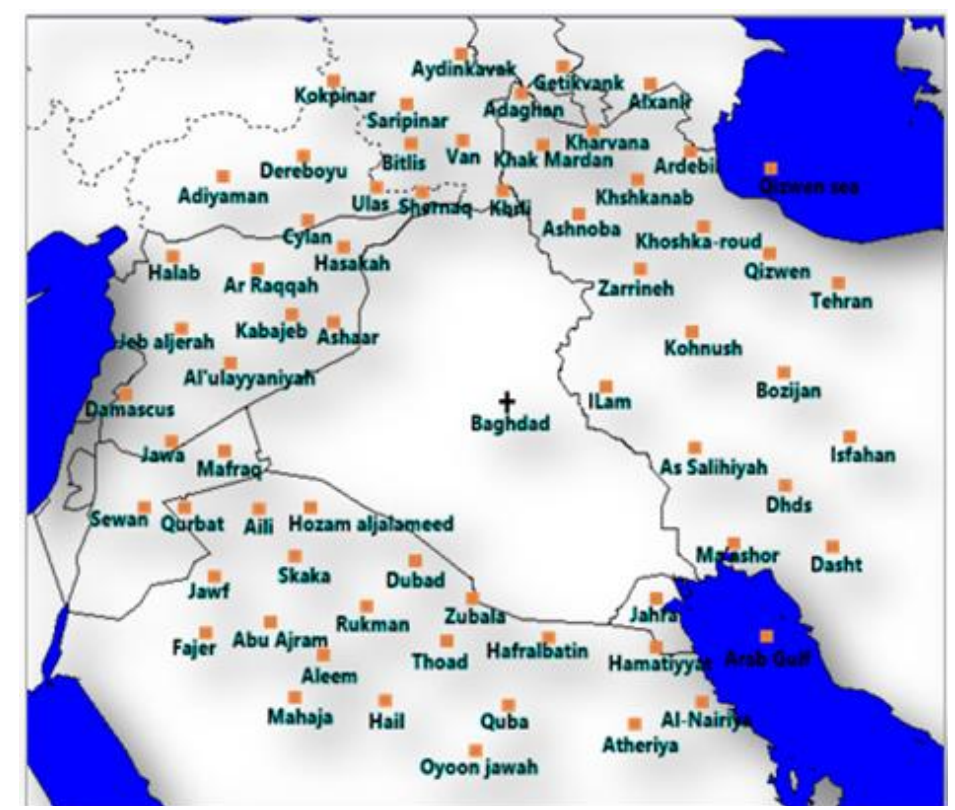

Figure 2:- The location of a transmitter and receiver stations over Middle East zone.

The datasets of the LUF ionospheric parameter have been determined using the REC533 international communication models, while the MUF and OWF parameters have been calculated using ASAPS international communication models. The monthly-observed sunspot number (SSN) for the years of 2009 and 2014 which have been chosen to be the studied years, because these years represent the beginning and the peak of solar cycle 24. Figure (3) shows the values of the observed sunspot number for each month of the selected years of solar cycle 24 .

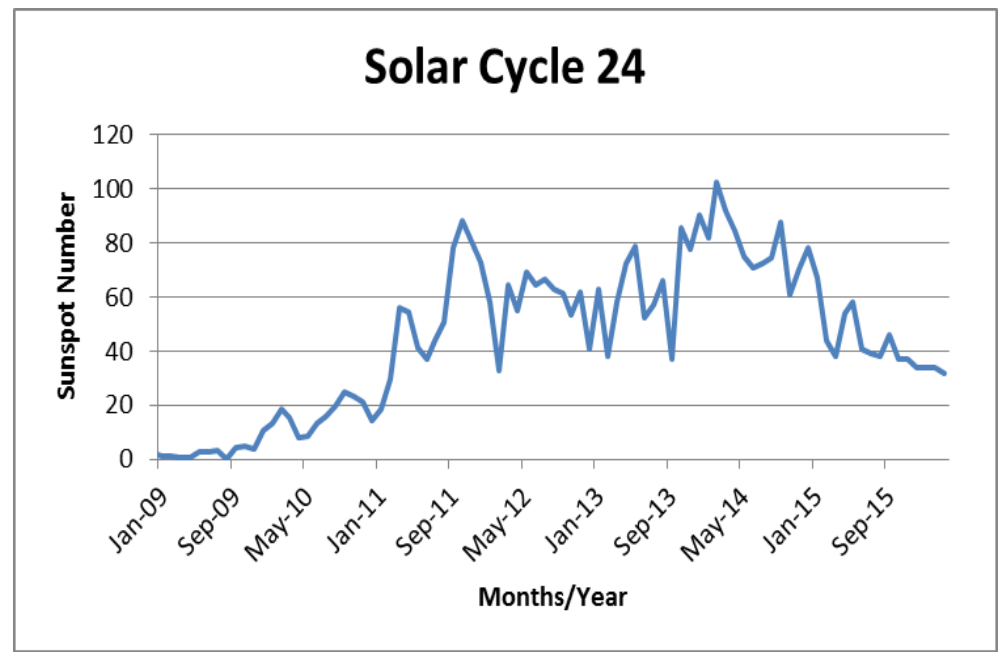

Figure 3:- Observed Sunspot Number of Solar Cycle 24 [7].

The geographical location coordinates (longitude and latitude), spherical geodesic parameters (path length - bearing transmitter to receiver $\left(T_{x}\right.$ to $\left.R_{x}\right)$ and bearing receiver to transmitter $\left(R_{x}\right.$ to $\left.T_{x}\right)$ ) and distance for connection links over the Middle East Region have been calculated using a matlab program language illustrate in table (1). 
Table 1:- The Geographical Location and Geodesic Parameters for all Receiver Station.

\begin{tabular}{|c|c|c|c|c|c|c|c|c|c|c|c|c|c|}
\hline \multirow[b]{2}{*}{ Receiver station } & \multicolumn{2}{|c|}{ Location } & \multirow{2}{*}{$\begin{array}{c}\text { Distance } \\
(\mathrm{Km})\end{array}$} & \multirow{2}{*}{$\begin{array}{l}\text { Path } \\
\text { length } \\
\text { (Rad) }\end{array}$} & \multicolumn{2}{|c|}{ Bearing } & \multirow[b]{2}{*}{ Receiver station } & \multicolumn{2}{|c|}{ Location } & \multirow{2}{*}{$\begin{array}{c}\text { Distance } \\
(\mathrm{Km})\end{array}$} & \multirow{2}{*}{$\begin{array}{c}\text { Path } \\
\text { length } \\
\text { (Rad) }\end{array}$} & \multicolumn{2}{|c|}{ Bearing } \\
\hline & $\begin{array}{c}\text { Long. } \\
\text { (E) }\end{array}$ & $\begin{array}{l}\text { Lat. } \\
\text { (N) }\end{array}$ & & & $\mathrm{Tx}$ to $\mathrm{Rx}$ & $\mathrm{Rx}$ to $\mathrm{Tx}$ & & $\begin{array}{c}\text { Long. } \\
\text { (E) }\end{array}$ & $\begin{array}{l}\text { Lat. } \\
\text { (N) }\end{array}$ & & & $\mathrm{Tx}$ to $\mathrm{Rx}$ & $\mathrm{Rx}$ to $\mathrm{Tx}$ \\
\hline Ilam & 46.240 & 33.380 & 169.170 & 0.027 & 88.180 & 272.320 & Halab & 37.800 & 36.120 & 680.220 & 0.107 & 117.120 & 297.120 \\
\hline Kharvana & 46.100 & 38.400 & 584.770 & 0.091 & 196.890 & 343.600 & ArRaqqah & 38.590 & 35.570 & 590.110 & 0.093 & 114930 & 294.930 \\
\hline Zarrineh & 47.100 & 36.400 & 420.760 & 0.066 & 143.800 & 323.800 & Damascus & 36.170 & 33.300 & 766.450 & 0.119 & 89.990 & 269990 \\
\hline Ashnoba & 45.510 & 37.200 & 442.630 & 0.069 & 166.520 & 346.520 & Jeb aljerah & 37.180 & 34.480 & 680.370 & 0.106 & 100.700 & 280.700 \\
\hline Kohnush & 48.160 & 34.430 & 366.640 & 0.058 & 71.370 & 252.420 & Al'ulayyaniyah & 38.350 & 33.500 & 563.690 & 0.088 & 91.700 & 271.700 \\
\hline Qizwen & 49.590 & 36.160 & 568.090 & 0.089 & 123.280 & 303.280 & Kabajeb & 39.400 & 35.400 & 515.480 & 0.081 & 113.860 & 295.280 \\
\hline Tehran & 51.250 & 35.410 & 668.400 & 0.105 & 109960 & 289960 & Jawa & 37900 & 32.350 & 618.520 & 0.096 & 100.400 & 280.400 \\
\hline Ardebil & 48.160 & 38.150 & 634.200 & 0.099 & 147.420 & 327.420 & AL-Qurbat & 37.220 & 31.190 & 717.080 & 0.112 & 109.630 & 289.630 \\
\hline As Salihiyah & 48.210 & 32.270 & 372.970 & 0.059 & 108.540 & 288.540 & Hozamjalamed & 40.600 & 31.160 & 432.100 & 0.067 & 124.410 & 304.410 \\
\hline Ma'ashor & 49.110 & 30.330 & 553.850 & 0.087 & 126920 & 306920 & Al Jawf & 38.100 & 29.570 & 730.030 & 0.114 & 125.210 & 305.210 \\
\hline Isfahan & 51.400 & 32.390 & 660.010 & 0.104 & 99.240 & 279.240 & AL-Dubad & 42.180 & 30.140 & 412.170 & 0.064 & 149.760 & 329.760 \\
\hline Dasht & 51.160 & 30.320 & 718.660 & 0.113 & 114910 & 296.690 & Hail & 41.410 & 27.300 & 728980 & 0.114 & 157.040 & 337.040 \\
\hline Bozijan & 50.140 & 33.550 & 531.320 & 0.083 & 92.380 & 272.380 & Abu Ajram & 39.140 & 29.100 & 687.050 & 0.107 & 133.490 & 313.490 \\
\hline Dhds & 50.170 & 31.420 & 579.760 & 0.091 & 111.540 & 291.540 & Hafralbatin & 45.120 & 28.430 & 547.820 & 0.086 & 187.660 & 352.520 \\
\hline Khoshlaa-roud & 48.310 & 36.480 & 499.220 & 0.078 & 134.160 & 314.160 & Quba & 44.200 & 27.240 & 676.390 & 0.106 & 178.040 & 270.170 \\
\hline Adaghan & 44.360 & 39.240 & 658.290 & 0.102 & 180.150 & 359.990 & Al-Hamatiyyat & 47.350 & 28.360 & 618320 & 0.097 & 150.890 & 241.650 \\
\hline Khshkanab & 47.700 & 37.420 & 544.230 & 0.085 & 146.370 & 326.370 & Al-Nairiya & 48.290 & 27.280 & 767350 & 0.120 & 148.710 & 239.700 \\
\hline KhakMardan & 45.400 & 38.230 & 553.060 & 0.086 & 170.370 & 350.370 & AL-Rukman & 41.160 & 29.200 & 552.990 & 0.086 & 146.450 & 326.450 \\
\hline Van & 43.220 & 38.290 & 563.110 & 0.087 & 169.210 & 349.210 & Skaka & 39.440 & 30.280 & 579.310 & 0.090 & 126.170 & 306.170 \\
\hline Khrli & 44.150 & 37.290 & 442.120 & 0.068 & 177.270 & 357.270 & Mahaja & 39.450 & 27.340 & 818.060 & 0.128 & 144.710 & 324.710 \\
\hline Saripinar & 42.800 & 39.120 & 661.060 & 0.103 & 167.540 & 347.540 & Oyoonjawah & 43.370 & 26.370 & 779.390 & 0.122 & 172.840 & 352.840 \\
\hline Kolkpinar & 40.340 & 39.390 & 767.270 & 0.120 & 148.230 & 329.450 & Fajer & 37.510 & 28.490 & 849940 & 0.133 & 129.510 & 309.510 \\
\hline Ceylan & 40.400 & 36.550 & 513.000 & 0.079 & 134.440 & 314.440 & Zubala & 43.320 & 29.320 & 456.870 & 0.072 & 167330 & 347330 \\
\hline Adiyaman & 38.120 & 37.460 & 733.200 & 0.114 & 128.860 & 308.860 & AL-Thoad & 43.000 & 28.400 & 563.590 & 0.088 & 163.810 & 255.180 \\
\hline Shernag & 42.270 & 37.300 & 483.600 & 0.076 & 153.610 & 334.230 & AL-Aleem & 40.230 & 28.240 & 692.140 & 0.108 & 145.100 & 325.100 \\
\hline Ulas & 41.320 & 37340 & 528.020 & 0.083 & 144.640 & 325.550 & Al-Atheriya & 47.200 & 27.200 & 730920 & 0.115 & 156.730 & 337.430 \\
\hline Bitlis & 42.150 & 38.250 & 585.120 & 0.091 & 159.740 & 339.740 & AL-Aili & 38.590 & 31.150 & 598960 & 0.093 & 114.190 & 294.190 \\
\hline Dereboyu & 39.560 & 38.100 & 689.000 & 0.107 & 140.520 & 320.520 & Jahra & 47.360 & 29.300 & 527.020 & 0.083 & 147.850 & 327.850 \\
\hline Getikvank & 45.300 & 39.560 & 698.290 & 0.110 & 172.620 & 352.890 & Mafraq & 38.180 & 32.250 & 595.230 & 0.092 & 101.910 & 281.910 \\
\hline Aydinkavak & 43.190 & 40.110 & 762.900 & 0.119 & 171970 & 351970 & Sewan & 36.340 & 31.160 & 796.710 & 0.124 & 107.850 & 287.850 \\
\hline Al Hasakah & 40.470 & 36.240 & 485.260 & 0.076 & 131980 & 311980 & Alxanli & 39.350 & 27.230 & 833.730 & 0.130 & 144.640 & 324.640 \\
\hline Ashaar & 40.330 & 34.550 & 401.300 & 0.062 & 109.650 & 289.650 & Arab Gulf & 49.510 & 28.470 & 725.520 & 0.114 & 135.140 & 226.460 \\
\hline
\end{tabular}

The seasonal variation of the ionospheric parameter has been investigated for four seasons. Each season represents a seasonal months for a specific studied area. For Middle East zone the seasonal months classified according to the following table (2).

Table 2:- The classification season time.

\begin{tabular}{|c|l|l|}
\hline & Season & \multicolumn{1}{c|}{ Months } \\
\hline 1 & Winter & December - January - February \\
\hline 2 & Spring & March - April - May \\
\hline 3 & Summer & June - July - August \\
\hline 4 & Autumn & September - October - November \\
\hline
\end{tabular}

The seasonal variation of the ionospheric parameters has been performed for all selected receiver stations over the tested area. Figures (4) \& (5) show the analytical result of the MUF, OWF and LUF parameters for seasonal time of the years 2009 and 2014. 

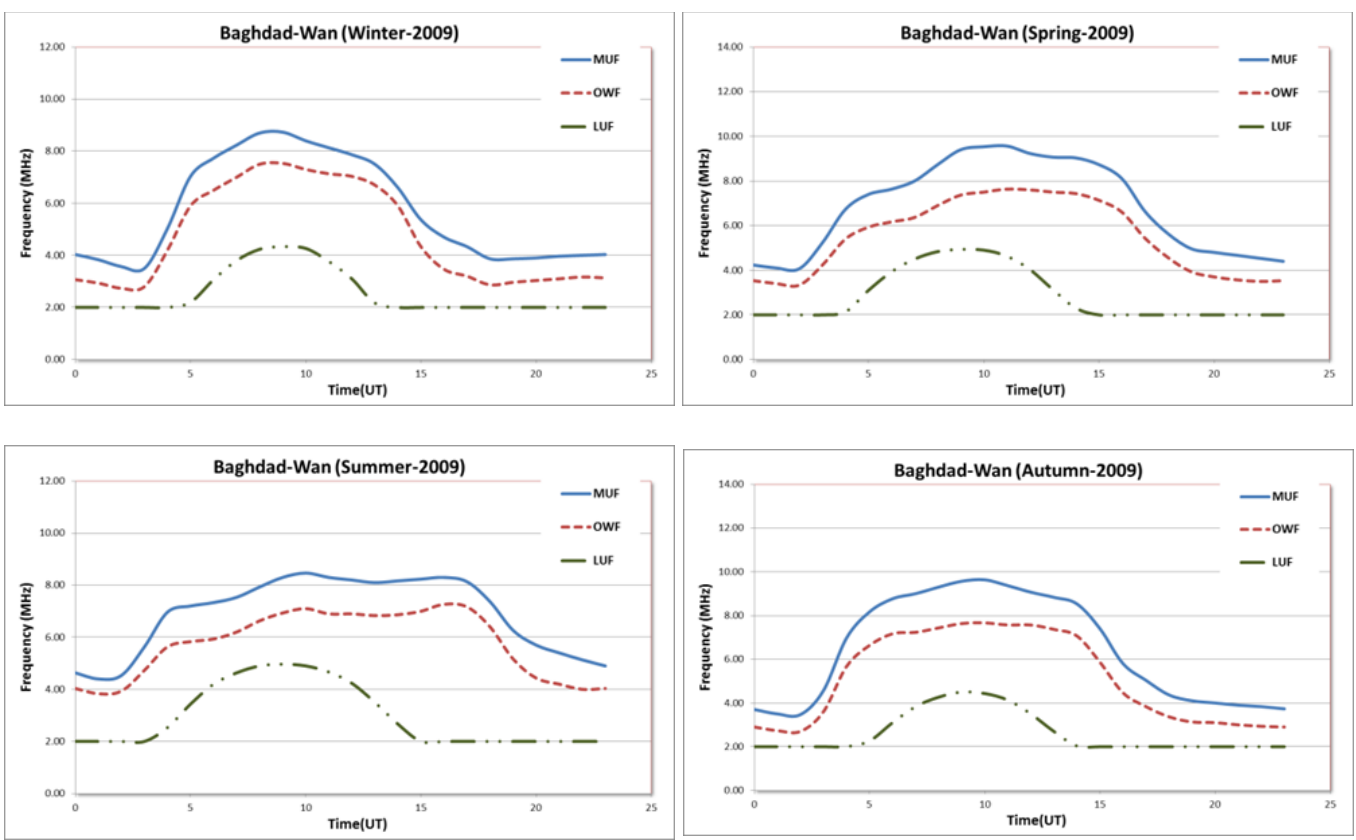

Figure 4:- Seasonal variation of ionospheric parameters (MUF, LUF \& OWF) for the year 2009.
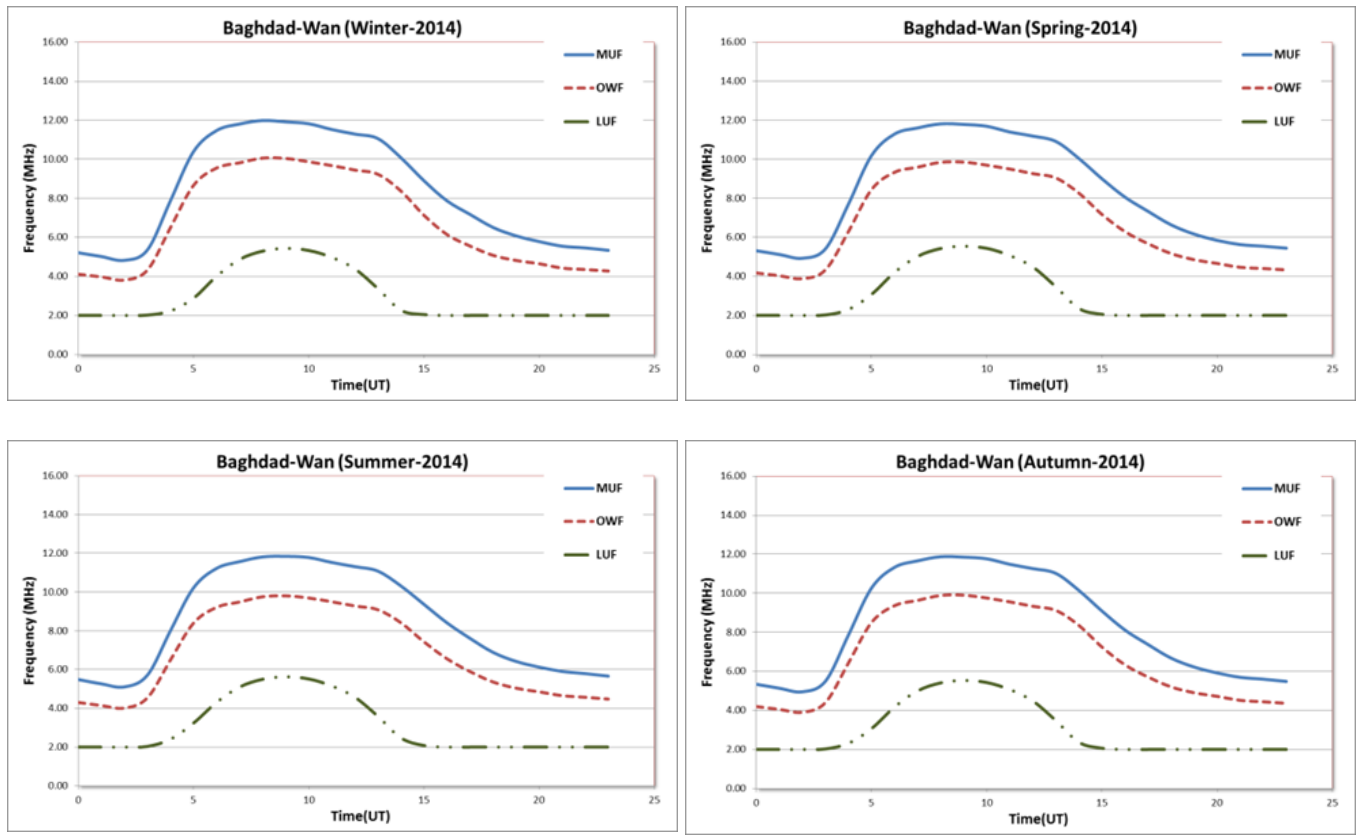

Figure 5:- Seasonal Variation of Ionospheric Parameters (MUF, LUF \& OWF) for the year 2014.

The goal of this research is to get a mutual correlation between the ionospheric parameters. In order to get these correlated relationships, an investigational study of the generated datasets has been conducted for the seasonal time of the two tested years. Table (3) show samples of the correlation coefficients $\left(a_{0}, a_{1}, a_{2}\right.$, $\left.a_{3} \& a_{4}\right)$ and correlation parameter $\left(R^{2}\right)$ over the Middle East zone of the seasonal time of the year 2009. 
Table 3:- Samples of the correlation coefficients and correlation parameter over the Middle East zone of the seasonal time of the year 2009.

\begin{tabular}{|c|c|c|c|c|c|c|}
\hline \multicolumn{7}{|c|}{ Baghdad- Wan (North Direction) } \\
\hline Winter & $\overline{a_{n}}$ & $\overline{a_{1}}$ & $\overline{a_{2}}$ & $\overline{a_{3}}$ & $\overline{a 4}$ & $\mathrm{R}^{2}$ \\
\hline MUF (OWF) & 0.800 & 1.037 & 0.000 & 0.000 & 0.000 & 0.993 \\
\hline MUF (LUF) & -360.160 & 466.770 & -217.070 & 43.951 & -3.273 & 0.889 \\
\hline OWF (MUF) & -0.737 & 0.958 & 0.000 & 0.000 & 0.000 & 0.993 \\
\hline OWF (LUF) & -352.320 & 455.430 & -211.720 & 42.848 & -3.190 & 0.870 \\
\hline LUF (MUF) & -19.237 & 15.135 & -3.831 & 0.402 & -0.014 & 0.964 \\
\hline LUF (OWF) & -2.704 & 3.701 & -0.944 & 0.079 & -0.000 & 0.929 \\
\hline Spring & $a_{0}$ & $\overline{a_{1}}$ & $a_{2}$ & $\overline{a 3}$ & $a_{4}$ & $R^{2}$ \\
\hline MUF (OWF) & 0.074 & 1.229 & 0.000 & 0.000 & 0.000 & 0.994 \\
\hline MUF (LUF) & -174.910 & 215.840 & -92.327 & 17.037 & -1.146 & 0.661 \\
\hline OWF (MUF) & -0.031 & 0.808 & 0.000 & 0.000 & 0.000 & 0.994 \\
\hline OWF (LUF) & -145.730 & 179.76 & - 76.988 & 14.237 & -0.961 & 0.633 \\
\hline LUF (MUF) & 62.917 & -39.451 & 9.274 & -0.940 & 0.035 & 0.614 \\
\hline LUF (OWF) & 37.370 & -26.194 & 6.942 & -0.785 & 0.032 & 0.522 \\
\hline Summer & $a_{0}$ & $a_{1}$ & $a_{2}$ & $a_{3}$ & $a_{4}$ & $R^{2}$ \\
\hline MUF (OWF) & 0.397 & 1.127 & 0.000 & 0.000 & 0.000 & 0.979 \\
\hline MUF (LUF) & -27.856 & 36.309 & -13.482 & 2.147 & -0.122 & 0.425 \\
\hline OWF (MUF) & -0.225 & 0.868 & 0.000 & 0.000 & 0.000 & 0.979 \\
\hline OWF (LUF) & -16.647 & 22.195 & -7.633 & 1.089 & -0.052 & 0.351 \\
\hline LUF (MUF) & 57.001 & -32.373 & 6.852 & -0.619 & 0.020 & 0.395 \\
\hline LUF (OWF) & -148.220 & 126.550 & -39.327 & 5.325 & -0.263 & 0.499 \\
\hline$\overline{\text { Autumn }}$ & $\overline{a_{0}}$ & $\overline{a_{1}}$ & $\overline{a_{2}}$ & $\overline{a 3}$ & $\overline{a 4}$ & $\mathrm{R}^{2}$ \\
\hline MUF (OWF) & 0.328 & 1.187 & 0.000 & 0.000 & 0.000 & 0.997 \\
\hline MUF (LUF) & -265.170 & 336.060 & -151.370 & 29.704 & -2.144 & 0.769 \\
\hline OWF (MUF) & -0.262 & 0.839 & 0.000 & 0.000 & 0.000 & 0.997 \\
\hline OWF (LUF) & -223.480 & 281.760 & -126.360 & 24.700 & -1.777 & 0.766 \\
\hline LUF (MUF) & 5.507 & -3.270 & 1.095 & -0.156 & 0.008 & 0.947 \\
\hline LUF (OWF) & 15.406 & -13.079 & 4.629 & -0.704 & 0.038 & 0.879 \\
\hline
\end{tabular}

The statistical analysis has been applied on the calculated datasets to assess the nature of correlation between these parameters. The results of the statistical analysis showed that the correlation between the ionospheric parameters could be expressed as a polynomial relationship, so the suggested mutual correlation equation between the studied parameters can be presented by the following equation:

$$
\begin{gathered}
Y=\sum_{i=0}^{n} a_{i} x^{i} \\
Y=a_{0}+a_{1} x^{1}+a_{2} x^{2}+a_{3} x^{3}+\cdots
\end{gathered}
$$

Figures (6) and (7) present illustrative charts of correlation between MUF and OWF parameters for all seasons of the years 2009 and 2014. 

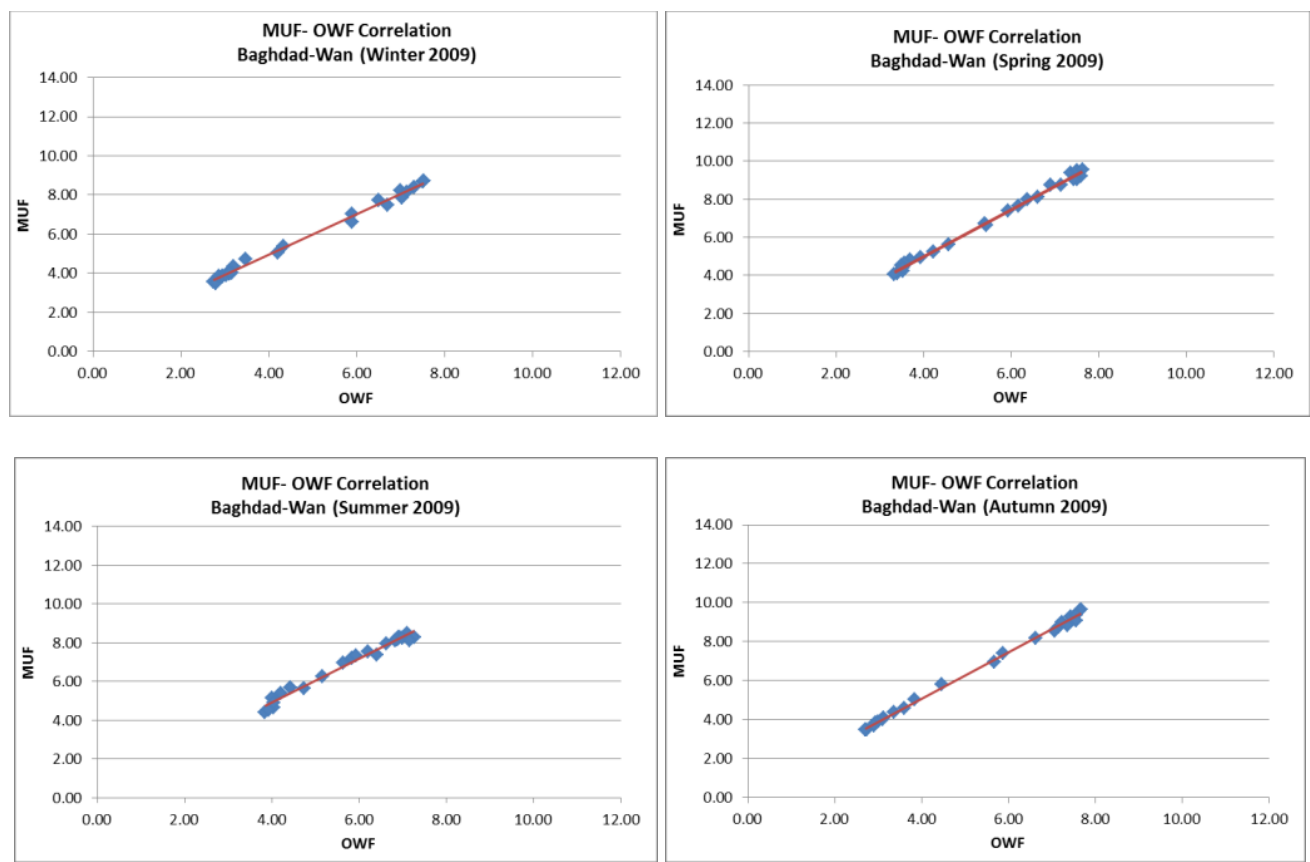

Figure 6:- Samples of correlation test between MUF and OWF parameters for seasonal time of year 2009.
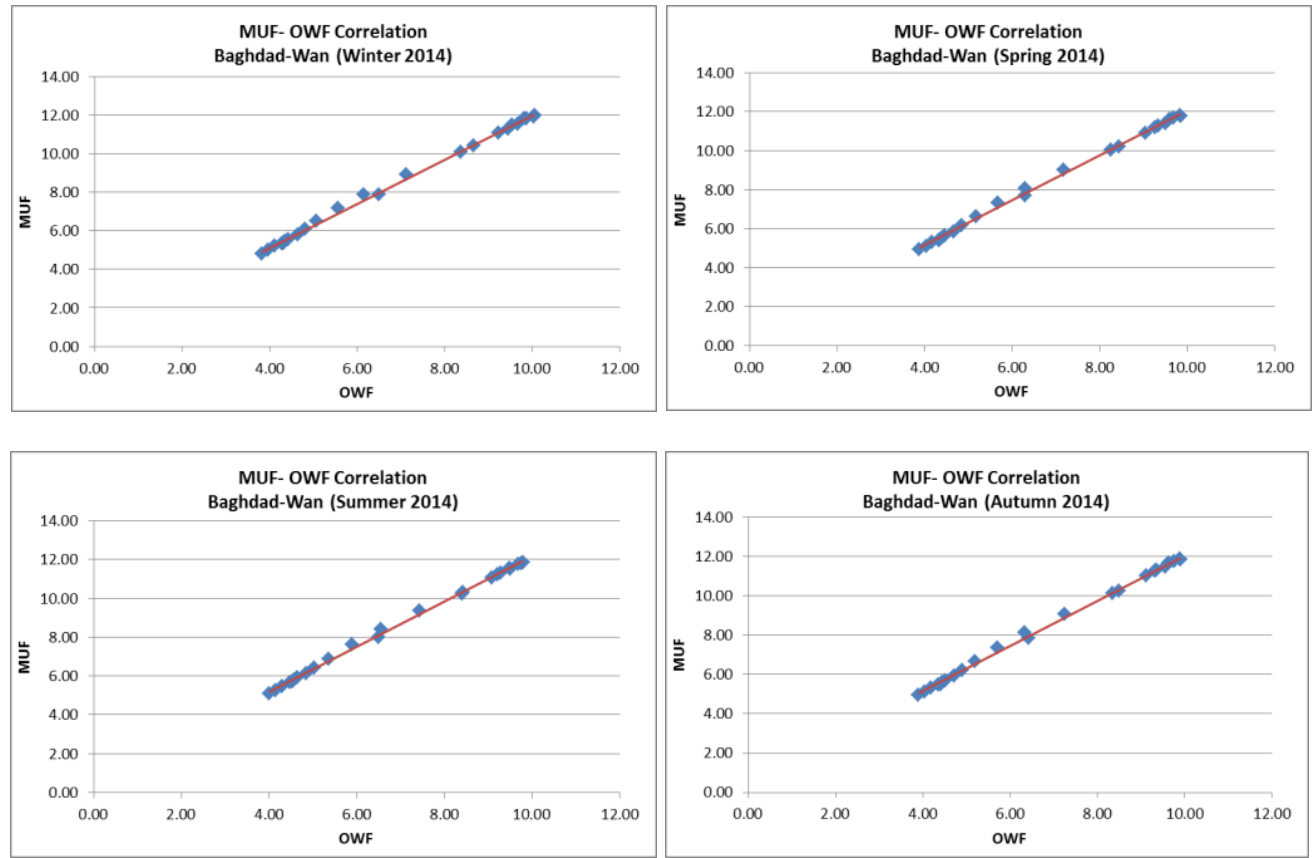

Figure 7:- Samples of the correlation between the MUF and OWF parameters for Seasonal times of the year 2014.

So, the suggested mutual correlation equations between (MUF, OWF and LUF), can be expressed by the following set of equations:

$$
\begin{aligned}
& \text { MUF }=\sum_{i=0}^{n} \mathrm{a}_{\mathrm{i}}(L U F)^{i} \\
& \mathrm{OWF}=\sum_{i=0}^{n} \mathrm{a}_{\mathrm{i}}(L U F)^{i}
\end{aligned}
$$


The values of MUF and OWF parameter have been correlated with LUF parameter for all distributed receiver locations over the adopted area for the seasonal time of the years 2009 and 2014. Figures (8) and (9)show samples of seasonal correlation between MUF and OWF with LUF parameters.

\section{(MUF-LUF)}
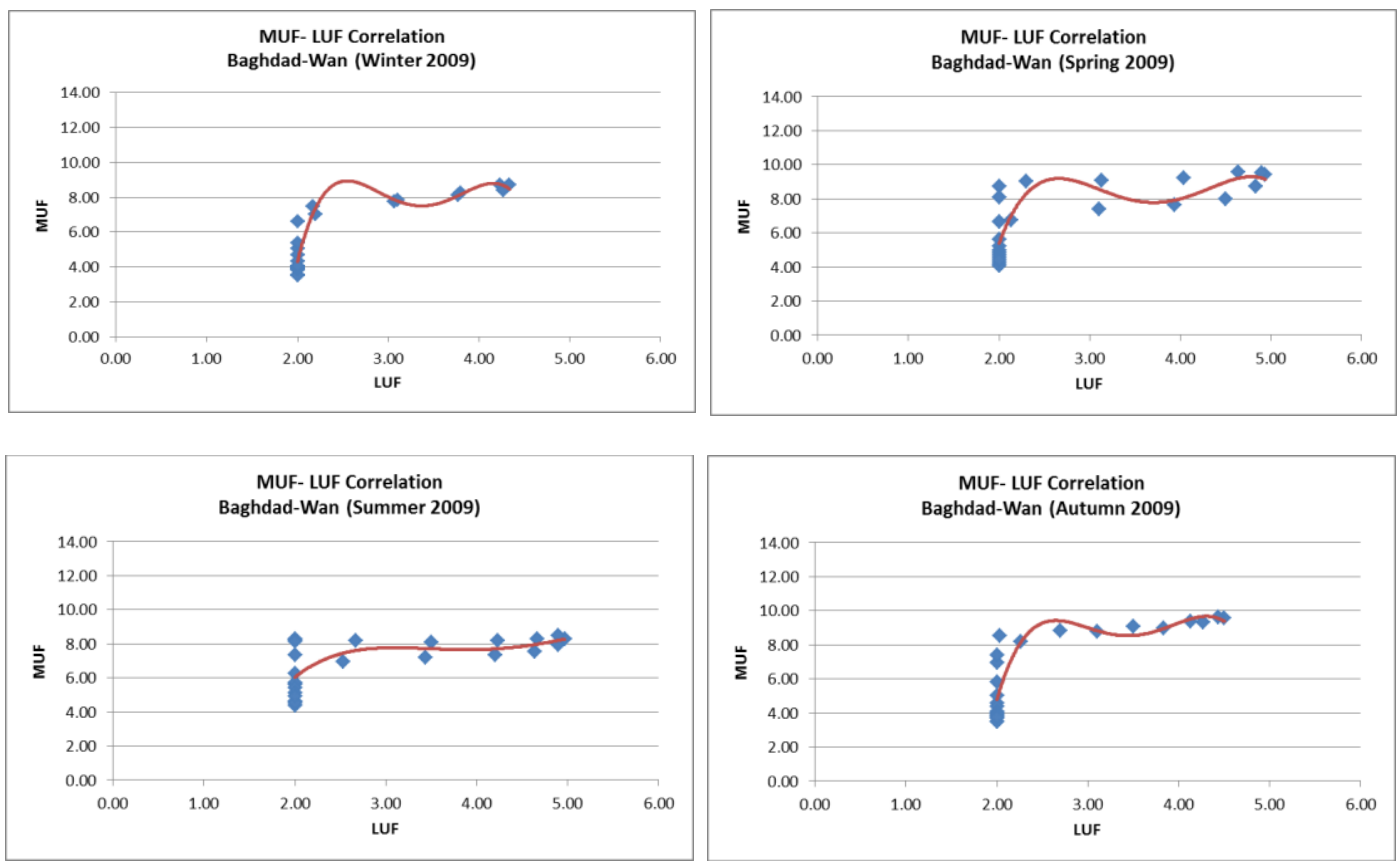

(OWF-LUF)
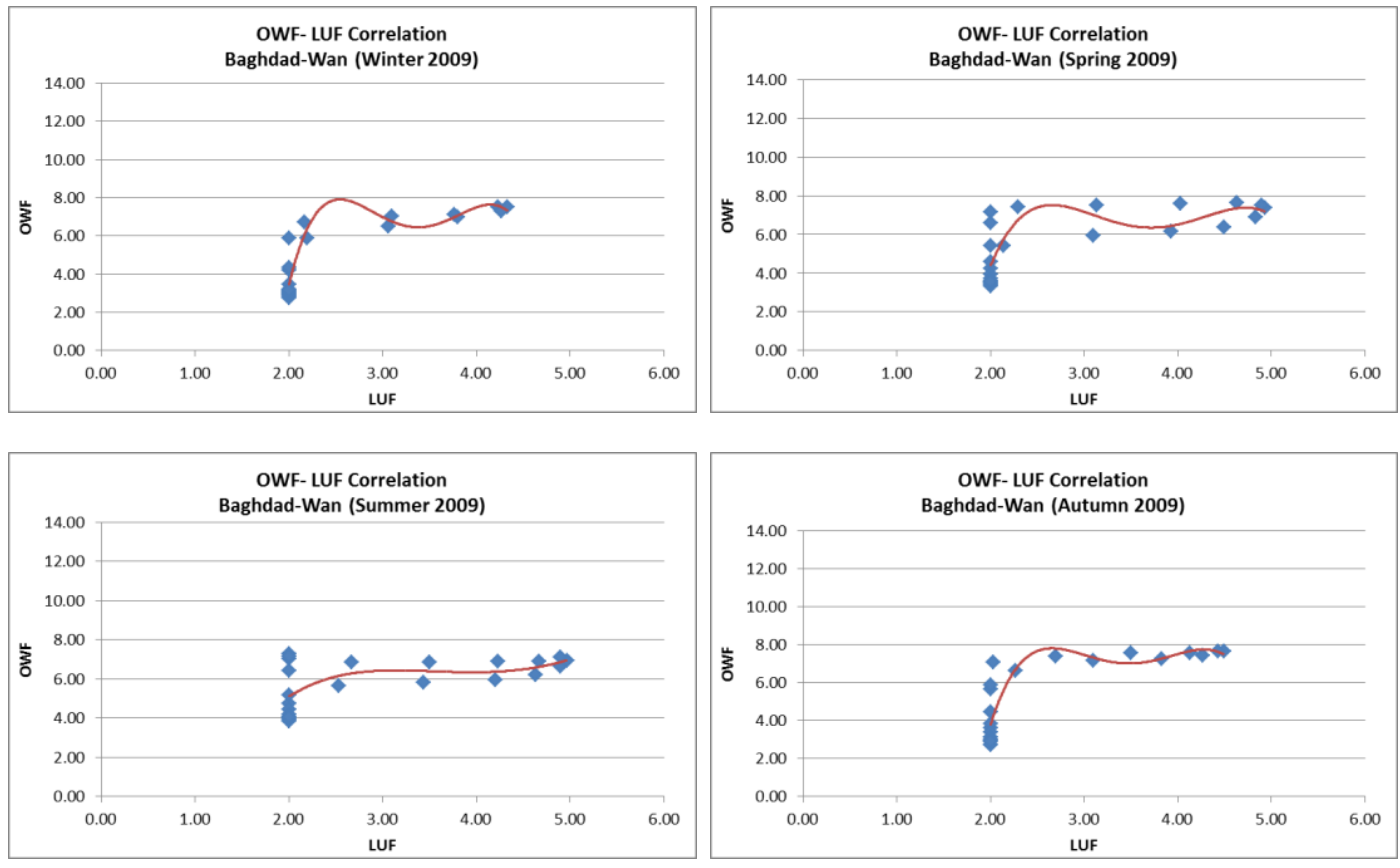

Figure 8:- Samples of the seasonal correlation between MUF, OWF with LUF parameters for the year 2009. 


\section{(MUF-LUF)}
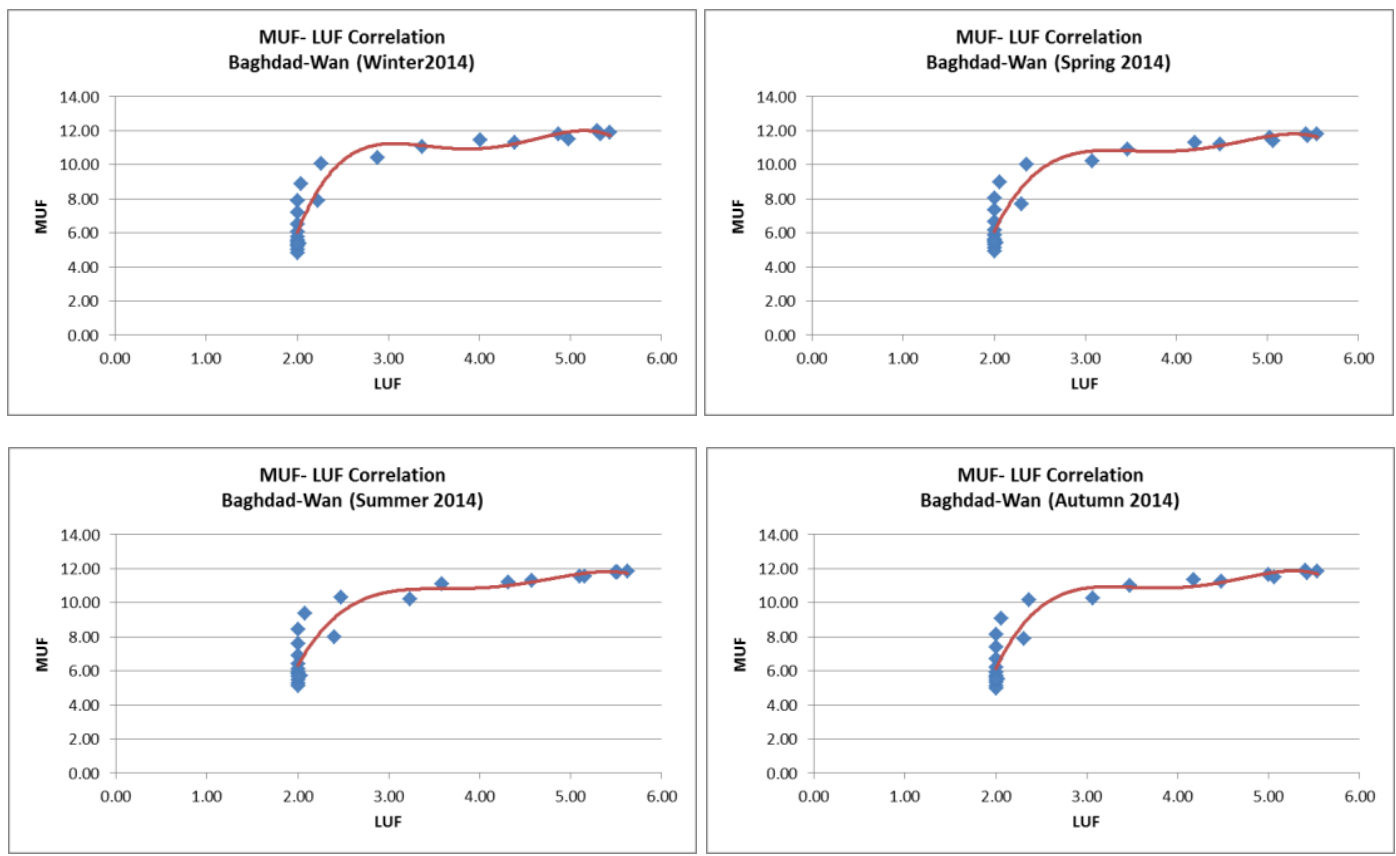

\section{(OWF-LUF)}
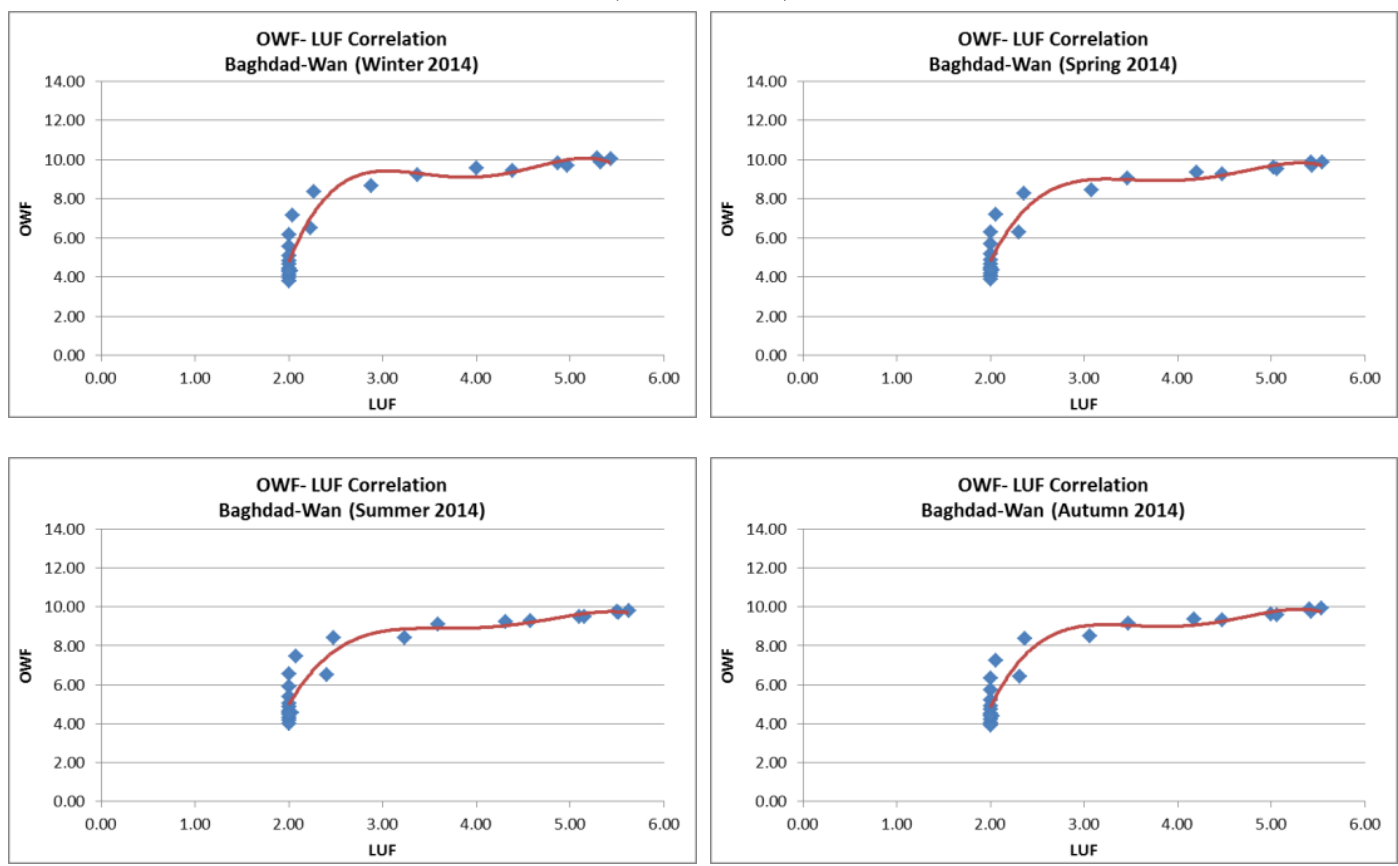

Figure 9:- Samples of seasonal correlation between the MUF, OWF with LUF parameters for year 2014

The comparison and mean square error (MSE) between the present value of MUF \& OWF ionospheric parameter that have been calculated using the suggested mutual correlated equations with the predicted ionospheric values that have been generated using the international model (ASAPS) and theoretical values calculated depending on the international criterion equation ( $\mathrm{OWF}=0.85 \mathrm{x}$ MUF) illustrate in table (4). 
Table 4:- The Seasonal Theoretical, Predicted \& Present value of the MUF, OWF parameters of adopted years.

\begin{tabular}{|c|c|c|c|c|c|c|c|c|c|c|c|c|}
\hline \multicolumn{10}{|c|}{ Winter Time } & & & \\
\hline \multicolumn{7}{|c|}{ Baghdad- Wan (MUF- 2009) } & \multicolumn{6}{|c|}{ Baghdad-Wan (MUF-2014) } \\
\hline \multirow{3}{*}{ UT } & \multirow{3}{*}{ OWF } & \multicolumn{5}{|c|}{ MUF } & \multirow{3}{*}{ OWF } & \multicolumn{5}{|c|}{ MUF } \\
\hline & & \multirow{2}{*}{ Predicted } & \multirow{2}{*}{ Theoretical } & \multirow{2}{*}{ Present } & \multicolumn{2}{|c|}{ MSE } & & \multirow{2}{*}{ Predicted } & \multirow{2}{*}{ Theoretical } & \multirow{2}{*}{ Present } & \multicolumn{2}{|c|}{ MSE } \\
\hline & & & & & Theo. & Pred. & & & & & Theo. & Pred. \\
\hline 0 & 3.07 & 4.03 & 3.61 & 3.98 & 0.139 & 0.003 & 4.11 & 5.21 & 4.83 & 5.24 & 0.167 & 0.001 \\
\hline 1 & 2.93 & 3.83 & 3.45 & 3.84 & 0.154 & 0.000 & 3.98 & 5.02 & 4.68 & 5.09 & 0.171 & 0.005 \\
\hline 2 & 2.73 & 3.57 & 3.22 & 3.64 & 0.176 & 0.005 & 3.81 & 4.82 & 4.49 & 4.91 & 0.175 & 0.007 \\
\hline 3 & 2.80 & 3.50 & 3.29 & 3.70 & 0.169 & 0.042 & 4.33 & 5.36 & 5.09 & 5.49 & 0.160 & 0.017 \\
\hline 4 & 4.20 & 5.03 & 4.94 & 5.16 & 0.047 & 0.015 & 6.50 & 7.88 & 7.65 & 7.97 & 0.104 & 0.008 \\
\hline 5 & 5.90 & 7.03 & 6.94 & 6.92 & 0.000 & 0.013 & 8.66 & 10.39 & 10.19 & 10.44 & 0.060 & 0.002 \\
\hline 6 & 6.50 & 7.73 & 7.65 & 7.54 & 0.011 & 0.036 & 9.55 & 11.47 & 11.24 & 11.45 & 0.046 & 0.000 \\
\hline 7 & 7.00 & 8.23 & 8.24 & 8.06 & 0.030 & 0.030 & 9.82 & 11.80 & 11.55 & 11.76 & 0.042 & 0.002 \\
\hline 8 & 7.50 & 8.70 & 8.82 & 8.58 & 0.059 & 0.014 & 10.06 & 11.98 & 11.83 & 12.03 & 0.038 & 0.002 \\
\hline 9 & 7.53 & 8.73 & 8.86 & 8.61 & 0.062 & 0.014 & 10.04 & 11.91 & 11.81 & 12.01 & 0.038 & 0.009 \\
\hline 10 & 7.30 & 8.40 & 8.59 & 8.37 & 0.047 & 0.001 & 9.87 & 11.82 & 11.61 & 11.82 & 0.041 & 0.000 \\
\hline 11 & 7.13 & 8.13 & 8.39 & 8.20 & 0.037 & 0.004 & 9.68 & 11.52 & 11.38 & 11.59 & 0.044 & 0.005 \\
\hline 12 & 7.03 & 7.87 & 8.27 & 8.10 & 0.032 & 0.053 & 9.45 & 11.29 & 11.11 & 11.33 & 0.047 & 0.002 \\
\hline 13 & 6.70 & 7.50 & 7.88 & 7.75 & 0.017 & 0.063 & 9.23 & 11.06 & 10.86 & 11.08 & 0.051 & 0.001 \\
\hline 14 & 5.90 & 6.60 & 6.94 & 6.92 & 0.000 & 0.103 & 8.37 & 10.08 & 9.85 & 10.11 & 0.065 & 0.001 \\
\hline 15 & 4.33 & 5.37 & 5.10 & 5.30 & 0.039 & 0.005 & 7.13 & 8.90 & 8.39 & 8.69 & 0.090 & 0.044 \\
\hline 16 & 3.47 & 4.70 & 4.08 & 4.40 & 0.101 & 0.092 & 6.14 & 7.87 & 7.23 & 7.56 & 0.113 & 0.093 \\
\hline 17 & 3.20 & 4.33 & 3.76 & 4.12 & 0.126 & 0.046 & 5.56 & 7.18 & 6.54 & 6.89 & 0.127 & 0.080 \\
\hline 18 & 2.87 & 3.87 & 3.37 & 3.77 & 0.161 & 0.009 & 5.08 & 6.51 & 5.97 & 6.35 & 0.140 & 0.025 \\
\hline 19 & 2.97 & 3.87 & 3.49 & 3.88 & 0.150 & 0.000 & 4.82 & 6.08 & 5.67 & 6.05 & 0.147 & 0.001 \\
\hline 20 & 3.03 & 3.90 & 3.57 & 3.95 & 0.143 & 0.002 & 4.65 & 5.78 & 5.47 & 5.86 & 0.151 & 0.005 \\
\hline 21 & 3.10 & 3.97 & 3.65 & 4.02 & 0.136 & 0.002 & 4.43 & 5.55 & 5.21 & 5.60 & 0.158 & 0.003 \\
\hline 22 & 3.17 & 4.00 & 3.73 & 4.08 & 0.129 & 0.007 & 4.35 & 5.46 & 5.12 & 5.52 & 0.160 & 0.004 \\
\hline 23 & 3.13 & 4.03 & 3.69 & 4.05 & 0.133 & 0.000 & 4.28 & 5.34 & 5.03 & 5.43 & 0.162 & 0.009 \\
\hline & & & Ave & MSE & 0.087 & 0.023 & & & Ave & e MSE & 0.104 & 0.014 \\
\hline
\end{tabular}

\begin{tabular}{|c|c|c|c|c|c|c|c|c|c|c|c|c|}
\hline \multicolumn{10}{|c|}{ Winter Time } & & & \\
\hline \multicolumn{7}{|c|}{ Baghdad- Wan (OWF- 2009) } & \multicolumn{6}{|c|}{ Baghdad-Wan (OWF- 2014) } \\
\hline \multirow{3}{*}{ UT } & \multirow{3}{*}{ MUF } & \multicolumn{5}{|c|}{ OWF } & \multirow{3}{*}{ MUF } & \multicolumn{5}{|c|}{ OWF } \\
\hline & & \multirow{2}{*}{ Predicted } & \multirow{2}{*}{ Theoretical } & \multirow{2}{*}{ Present } & \multicolumn{2}{|c|}{ MSE } & & \multirow{2}{*}{ Predicted } & \multirow{2}{*}{ Theoretical } & \multirow{2}{*}{ Present } & \multicolumn{2}{|c|}{ MSE } \\
\hline & & & & & Theo. & Pred. & & & & & Theo. & Pred. \\
\hline 0 & 4.03 & 3.07 & 3.43 & 3.13 & 0.091 & 0.004 & 5.21 & 4.11 & 4.43 & 4.09 & 0.118 & 0.000 \\
\hline 1 & 3.83 & 2.93 & 3.26 & 2.94 & 0.104 & 0.000 & 5.02 & 3.98 & 4.26 & 3.92 & 0.121 & 0.003 \\
\hline 2 & 3.57 & 2.73 & 3.03 & 2.68 & 0.124 & 0.003 & 4.82 & 3.81 & 4.10 & 3.75 & 0.124 & 0.005 \\
\hline 3 & 3.50 & 2.80 & 2.98 & 2.62 & 0.129 & 0.034 & 5.36 & 4.33 & 4.55 & 4.22 & 0.115 & 0.012 \\
\hline 4 & 5.03 & 4.20 & 4.28 & 4.09 & 0.037 & 0.013 & 7.88 & 6.50 & 6.70 & 6.42 & 0.076 & 0.006 \\
\hline 5 & 7.03 & 5.90 & 5.98 & 6.00 & 0.001 & 0.010 & 10.39 & 8.66 & 8.83 & 8.62 & 0.046 & 0.002 \\
\hline 6 & 7.73 & 6.50 & 6.57 & 6.67 & 0.010 & 0.030 & 11.47 & 9.55 & 9.75 & 9.56 & 0.035 & 0.000 \\
\hline 7 & 8.23 & 7.00 & 7.00 & 7.15 & 0.023 & 0.023 & 11.80 & 9.82 & 10.03 & 9.85 & 0.032 & 0.001 \\
\hline 8 & 8.70 & 7.50 & 7.40 & 7.60 & 0.041 & 0.010 & 11.98 & 10.06 & 10.19 & 10.01 & 0.030 & 0.002 \\
\hline 9 & 8.73 & 7.53 & 7.42 & 7.63 & 0.043 & 0.009 & 11.91 & 10.04 & 10.13 & 9.95 & 0.031 & 0.008 \\
\hline 10 & 8.40 & 7.30 & 7.14 & 7.31 & 0.029 & 0.000 & 11.82 & 9.87 & 10.04 & 9.87 & 0.032 & 0.000 \\
\hline 11 & 8.13 & 7.13 & 6.91 & 7.06 & 0.020 & 0.006 & 11.52 & 9.68 & 9.79 & 9.61 & 0.034 & 0.004 \\
\hline 12 & 7.87 & 7.03 & 6.69 & 6.80 & 0.013 & 0.055 & 11.29 & 9.45 & 9.60 & 9.40 & 0.037 & 0.002 \\
\hline 13 & 7.50 & 6.70 & 6.38 & 6.45 & 0.005 & 0.063 & 11.06 & 9.23 & 9.40 & 9.20 & 0.039 & 0.001 \\
\hline 14 & 6.60 & 5.90 & 5.61 & 5.59 & 0.001 & 0.099 & 10.08 & 8.37 & 8.57 & 8.34 & 0.049 & 0.001 \\
\hline 15 & 5.37 & 4.33 & 4.56 & 4.40 & 0.025 & 0.005 & 8.90 & 7.13 & 7.57 & 7.31 & 0.063 & 0.034 \\
\hline 16 & 4.70 & 3.47 & 4.00 & 3.77 & 0.053 & 0.089 & 7.87 & 6.14 & 6.69 & 6.41 & 0.077 & 0.072 \\
\hline 17 & 4.33 & 3.20 & 3.68 & 3.41 & 0.072 & 0.046 & 7.18 & 5.56 & 6.10 & 5.80 & 0.086 & 0.062 \\
\hline 18 & 3.87 & 2.87 & 3.29 & 2.97 & 0.102 & 0.010 & 6.51 & 5.08 & 5.53 & 5.22 & 0.097 & 0.020 \\
\hline 19 & 3.87 & 2.97 & 3.29 & 2.97 & 0.102 & 0.000 & 6.08 & 4.82 & 5.16 & 4.84 & 0.103 & 0.001 \\
\hline 20 & 3.90 & 3.03 & 3.32 & 3.00 & 0.100 & 0.001 & 5.78 & 4.65 & 4.92 & 4.59 & 0.108 & 0.004 \\
\hline 21 & 3.97 & 3.10 & 3.37 & 3.06 & 0.095 & 0.001 & 5.55 & 4.43 & 4.72 & 4.38 & 0.112 & 0.002 \\
\hline 22 & 4.00 & 3.17 & 3.40 & 3.10 & 0.093 & 0.005 & 5.46 & 4.35 & 4.64 & 4.30 & 0.114 & 0.003 \\
\hline 23 & 4.03 & 3.13 & 3.43 & 3.13 & 0.091 & 0.000 & 5.34 & 4.28 & 4.54 & 4.20 & 0.116 & 0.006 \\
\hline & & & Ave & MSE & 0.058 & 0.022 & & & Ave & MSE & 0.075 & 0.010 \\
\hline
\end{tabular}




\begin{tabular}{|c|c|c|c|c|c|c|c|c|c|c|c|c|}
\hline \multicolumn{10}{|c|}{ Summer Time } & & & \\
\hline \multirow{4}{*}{ UT } & \multicolumn{6}{|c|}{ Baghdad-Wan (OWF- 2009) } & \multicolumn{6}{|c|}{ Baghdad-Wan (OWF- 2014) } \\
\hline & \multirow{3}{*}{ MUF } & \multicolumn{5}{|c|}{ OWF } & \multirow{3}{*}{ MUF } & \multicolumn{5}{|c|}{ OWF } \\
\hline & & \multirow{2}{*}{ Predicted } & \multirow{2}{*}{ Theoretical } & \multirow{2}{*}{ Present } & \multicolumn{2}{|c|}{ MSE } & & \multirow{2}{*}{ Predicted } & \multirow{2}{*}{ Theoretical } & \multirow{2}{*}{ Present } & \multicolumn{2}{|c|}{ MSE } \\
\hline & & & & & Theo. & Pred. & & & & & Theo. & Pred. \\
\hline 0 & 4.63 & 4.03 & 3.94 & 3.80 & 0.020 & 0.056 & 5.49 & 4.30 & 4.66 & 4.27 & 0.153 & 0.001 \\
\hline 1 & 4.40 & 3.83 & 3.74 & 3.59 & 0.021 & 0.057 & 5.26 & 4.14 & 4.47 & 4.08 & 0.154 & 0.004 \\
\hline 2 & 4.53 & 3.93 & 3.85 & 3.71 & 0.020 & 0.050 & 5.11 & 4.01 & 4.34 & 3.95 & 0.155 & 0.005 \\
\hline 3 & 5.63 & 4.73 & 4.79 & 4.67 & 0.015 & 0.005 & 5.71 & 4.54 & 4.86 & 4.47 & 0.151 & 0.006 \\
\hline 4 & 6.97 & 5.63 & 5.92 & 5.82 & 0.010 & 0.036 & 8.01 & 6.50 & 6.81 & 6.44 & 0.137 & 0.004 \\
\hline 5 & 7.20 & 5.83 & 6.12 & 6.03 & 0.009 & 0.037 & 10.22 & 8.40 & 8.69 & 8.33 & 0.124 & 0.004 \\
\hline 6 & 7.33 & .93 & 6.23 & 6.14 & 0.008 & 0.043 & 11.22 & 9.21 & 9.54 & 9.20 & 0.119 & 0.000 \\
\hline 7 & 7.53 & 6.20 & 6.40 & 6.32 & 0.008 & 0.013 & 11.57 & 9.49 & 9.84 & 9.49 & 0.117 & 0.000 \\
\hline 8 & \begin{tabular}{|l|}
7.93 \\
\end{tabular} & 6.63 & 6.74 & 6.66 & 0.007 & 0.001 & 11.81 & 9.76 & 10.04 & 9.70 & 0.115 & 0.003 \\
\hline 9 & 8.30 & 6.93 & 7.06 & 6.98 & 0.005 & 0.002 & 11.85 & 9.81 & 10.07 & 9.73 & 0.115 & 0.006 \\
\hline 10 & 8.47 & 7.10 & 7.20 & 7.13 & 0.005 & 0.001 & 11.79 & 9.70 & 10.02 & 9.68 & 0.116 & 0.000 \\
\hline 11 & 8.30 & 6.90 & 7.06 & 6.98 & 0.005 & 0.007 & 11.53 & 9.50 & 9.80 & 9.46 & 0.117 & 0.002 \\
\hline 12 & 8.20 & 6.90 & 6.97 & 6.89 & 0.006 & 0.000 & 11.31 & 9.28 & 9.62 & 9.27 & 0.118 & 0.000 \\
\hline 13 & 8.10 & 6.83 & 6.89 & 6.81 & 0.006 & 0.001 & 11.08 & 9.09 & 9.42 & 9.07 & 0.120 & 0.000 \\
\hline 14 & 8.17 & 6.87 & 6.94 & 6.87 & 0.006 & 0.000 & 10.32 & 8.42 & 8.77 & 8.42 & 0.124 & 0.000 \\
\hline 15 & 8.23 & & & 6.92 & 0.006 & 0.006 & 9.37 & 7.43 & 7.96 & 7.60 & 0.129 & 0.029 \\
\hline 16 & 8.30 & 27 & 7.06 & 6.98 & 0.005 & 0.082 & 8.41 & 6.56 & 7.15 & 6.78 & 0.135 & 0.051 \\
\hline 17 & 8.13 & .17 & 6.91 & 6.84 & 0.006 & 0.109 & 7.61 & 5.90 & 6.47 & 6.10 & 0.140 & 0.039 \\
\hline 18 & 7.37 & 6.40 & 6.26 & 6.17 & 0.008 & 0.053 & 6.89 & 5.36 & 5.85 & 5.47 & 0.144 & 0.013 \\
\hline 19 & 6.27 & 5.17 & 5.33 & 5.22 & 0.012 & 0.002 & 6.42 & 5.04 & 5.46 & 5.08 & 0.147 & 0.001 \\
\hline 20 & 5.70 & 4.43 & 4.85 & 4.72 & 0.015 & 0.084 & 6.13 & 4.85 & 5.21 & 4.82 & 0.149 & 0.001 \\
\hline 21 & 5.40 & & & 4.46 & 0.016 & 0.069 & 5.91 & 4.66 & 5.02 & 4.63 & 0.150 & 0.001 \\
\hline 22 & 5.13 & 4.00 & 4.36 & 4.23 & 0.017 & 0.054 & 5.79 & 4.57 & 4.92 & 4.54 & 0.151 & 0.001 \\
\hline 23 & 4.90 & 4.03 & 4.17 & 4.03 & 0.019 & 0.000 & 5.66 & 4.48 & 4.81 & 4.42 & 0.152 & 0.003 \\
\hline & & & Av & MSE & 0.011 & 0.032 & & & Ave & MSE & 0.135 & 0.007 \\
\hline
\end{tabular}

\begin{tabular}{|c|c|c|c|c|c|c|c|c|c|c|c|c|}
\hline \multicolumn{10}{|c|}{ Summer Time } & & & \\
\hline \multicolumn{7}{|c|}{ Baghdad-Wan (MUF- 2009) } & \multicolumn{6}{|c|}{ Baghdad-Wan (MUF-2014) } \\
\hline \multirow{3}{*}{ UT } & \multirow{3}{*}{ OWF } & \multicolumn{5}{|c|}{ MUF } & \multirow{3}{*}{ OWF } & \multicolumn{5}{|c|}{ MUF } \\
\hline & & \multirow{2}{*}{ Predicted } & \multirow{2}{*}{ Theoretical } & \multirow{2}{*}{ Present } & \multicolumn{2}{|c|}{ MSE } & & \multirow{2}{*}{ Predicted } & \multirow{2}{*}{ Theoretical } & \multirow{2}{*}{ Present } & \multicolumn{2}{|c|}{ MSE } \\
\hline & & & & & Theo. & Pred. & & & & & Theo. & Pred. \\
\hline 0 & 4.03 & 4.63 & 4.75 & 4.95 & 0.040 & 0.098 & 4.30 & 5.49 & 5.06 & 5.53 & 0.216 & 0.002 \\
\hline 1 & 3.83 & 4.40 & 4.51 & 4.72 & 0.044 & 0.103 & 4.14 & 5.26 & 4.88 & 5.34 & 0.217 & 0.006 \\
\hline 2 & 3.93 & 4.53 & 4.63 & 4.83 & 0.042 & 0.090 & 4.01 & 5.11 & 4.72 & 5.19 & 0.219 & 0.007 \\
\hline 3 & 4.73 & 5.63 & 5.57 & 5.74 & 0.028 & 0.010 & 4.54 & 5.71 & 5.35 & 5.81 & 0.213 & 0.009 \\
\hline 4 & 5.63 & 6.97 & 6.63 & 6.75 & 0.015 & 0.047 & 6.50 & 8.01 & 7.65 & 8.08 & 0.190 & 0.005 \\
\hline 5 & 5.83 & 7.20 & 6.86 & 6.98 & 0.013 & 0.050 & 8.40 & 10.22 & 9.88 & 10.29 & 0.169 & 0.005 \\
\hline 6 & 5.93 & 7.33 & 6.98 & 7.09 & 0.012 & 0.060 & 9.21 & 11.22 & 10.84 & 11.24 & 0.161 & 0.000 \\
\hline 7 & 6.20 & 7.53 & 7.29 & 7.39 & 0.009 & 0.021 & 9.49 & 11.57 & 11.16 & 11.56 & 0.158 & 0.000 \\
\hline 8 & 6.63 & 7.93 & 7.80 & 7.88 & 0.006 & 0.003 & 9.76 & 11.81 & 11.48 & 11.88 & 0.155 & 0.004 \\
\hline 9 & 6.93 & 8.30 & 8.16 & 8.22 & 0.004 & 0.007 & 9.81 & 11.85 & 11.54 & 11.93 & 0.155 & 0.007 \\
\hline 10 & 7.10 & 8.47 & 8.35 & 8.40 & 0.003 & 0.004 & 9.70 & 11.79 & 11.41 & 11.80 & 0.156 & 0.000 \\
\hline 11 & 6.90 & 8.30 & 8.12 & 8.18 & 0.004 & 0.015 & 9.50 & 11.53 & 11.18 & 11.57 & 0.158 & 0.002 \\
\hline 12 & 6.90 & 8.20 & 8.12 & 8.18 & 0.004 & 0.000 & 9.28 & 11.31 & 10.92 & 11.32 & 0.160 & 0.000 \\
\hline 13 & 6.83 & 8.10 & 8.04 & 8.10 & 0.004 & 0.000 & 9.09 & 11.08 & 10.69 & 11.09 & 0.162 & 0.000 \\
\hline 14 & 6.87 & 8.17 & 8.08 & 8.14 & 0.004 & 0.001 & 8.42 & 10.32 & 9.90 & 10.31 & 0.169 & 0.000 \\
\hline 15 & 7.00 & 8.23 & 8.24 & 8.29 & 0.003 & 0.003 & 7.43 & 9.37 & 8.74 & 9.17 & 0.180 & 0.040 \\
\hline 16 & 7.27 & 8.30 & 8.55 & 8.59 & 0.002 & 0.086 & 6.56 & 8.41 & 7.71 & 8.15 & 0.189 & 0.069 \\
\hline 17 & 7.17 & 8.13 & 8.43 & 8.48 & 0.002 & 0.120 & 5.90 & 7.61 & 6.94 & 7.38 & 0.197 & 0.052 \\
\hline 18 & 6.40 & 7.37 & 7.53 & 7.62 & 0.007 & 0.062 & 5.36 & 6.89 & 6.31 & 6.76 & 0.203 & 0.017 \\
\hline 19 & 5.17 & 6.27 & 6.08 & 6.22 & 0.021 & 0.002 & 5.04 & 6.42 & 5.93 & 6.39 & 0.207 & 0.001 \\
\hline 20 & 4.43 & 5.70 & 5.22 & 5.40 & 0.033 & 0.092 & 4.85 & 6.13 & 5.71 & 6.16 & 0.209 & 0.001 \\
\hline 21 & 4.20 & 5.40 & 4.94 & 5.13 & 0.037 & 0.071 & 4.66 & 5.91 & 5.48 & 5.94 & 0.211 & 0.001 \\
\hline 22 & 4.00 & 5.13 & 4.71 & 4.91 & 0.041 & 0.051 & 4.57 & 5.79 & 5.38 & 5.84 & 0.212 & 0.002 \\
\hline 23 & 4.03 & 4.90 & 4.75 & 4.95 & 0.040 & 0.002 & 4.48 & 5.66 & 5.27 & 5.73 & 0.214 & 0.004 \\
\hline & & & Ave & ISE & 0.017 & 0.041 & & & Ave & MSE & 0.187 & 0.010 \\
\hline
\end{tabular}




\section{Conclusion:-}

1. For the determination of bearing parameter, the first method showed more accurate values than the second one.

2. The behavior of the ionospheric parameters for the seasonal times showed a variation in the values of these parameters for the two studied years, especially for the summer time, which may due to the impact of the solar activity (sunspot number) on the structure of the ionosphere layer (electron density).

3. The mutual correlation between MUF \& OWF is simple and can be represented by a simple mathematical relationship "linear regression equation".

4. The values calculated from the suggested mutual correlation equation gave a good fit with the other values generated from the international model and criterion.

5. The correlation between MUF and OWF parameters achieved a percentage mutuality of $99 \%$ (correlated coefficient) that gave better correlated mutuality than the international recommended criterion $(85 \%)$.

6. The correlated relationship between (MUF \& LUF), (OWF \& LUF) is a polynomial which represented by a fourth order polynomial equation (Quartic Polynomial Equation).

7. The datasets which have been generated from the suggested mutual correlation equation was closer to the values calculated from the international model than the values calculated depending on the international recommended criterion.

\section{References:-}

1. Alam, S. N. , Khan, M. A., Jilani, A. A. and Mian, K. (2012).Investigating the Fluctuating Dynamics of Refractive Index of Ionospheric Layer at Pakistan Region. (2012). Journal of Information \& Communication Technology. 6: 40-47.

2. Introduction to HF Radio Propagation. (2000). IPS Radio and Space Service. Australian Government.

3. Norm, C. and Kenneth, D. (1994). Radio Wave Propagation. Space Environment Laboratory. NOAA. Boulder. pp.1

4. Golygin, V. A., Vdovin, E. M., Sazhin, V. I. \&Unuchkov, V. E. (2009). Adaptation of the average monthly ionospheric model to current conditions using the data on maximum usable frequencies at twohop reference radio links. Geomagnetism and Aeronomy. 49: 368-373.

5. Douglas, J. and Boore, D. M. (2011). High- frequency filtering of strong- motion records. Bulletin of Earthquake Engineering. 9: 395-409.

6. Sailors, D. B. and Rose, R. B. (1993). HF Sky-Wave Field Strength Predictions. Naval Command. Control and Ocean Surveillance Center RDT\&E Division. San Diego. California. Technical Report 1624.

7. Monthly Sunspot Numbers. (2016). http://www.sws.bom.gov.au/Solar/1/6. Bureau of Meteorology.Australain Government. 